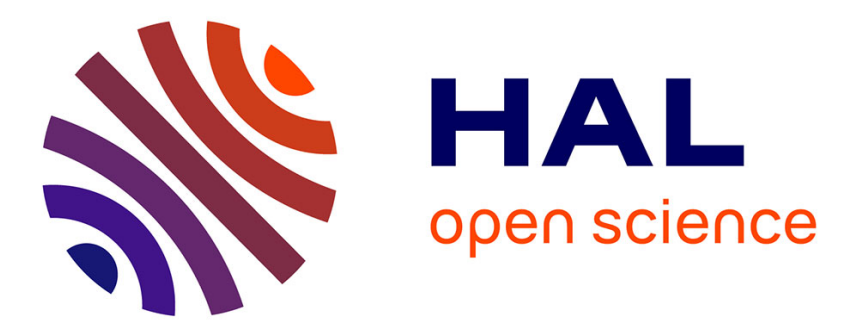

\title{
Performances analysis of piezoelectric cantilever based energy harvester devoted to mesoscale intra-body robot.
}

Kanty Rabenorosoa, Micky Rakotondrabe

\section{To cite this version:}

Kanty Rabenorosoa, Micky Rakotondrabe. Performances analysis of piezoelectric cantilever based energy harvester devoted to mesoscale intra-body robot.. Sensors for Next-Generation Robotics, Apr 2016, Baltimore, United States. hal-02868230

\section{HAL Id: hal-02868230 \\ https://hal.science/hal-02868230}

Submitted on 15 Jun 2020

HAL is a multi-disciplinary open access archive for the deposit and dissemination of scientific research documents, whether they are published or not. The documents may come from teaching and research institutions in France or abroad, or from public or private research centers.
L'archive ouverte pluridisciplinaire HAL, est destinée au dépôt et à la diffusion de documents scientifiques de niveau recherche, publiés ou non, émanant des établissements d'enseignement et de recherche français ou étrangers, des laboratoires publics ou privés. 


\title{
Performances analysis of piezoelectric cantilever based energy harvester devoted to mesoscale intra-body robot
}

\author{
Kanty Rabenorosoa and Micky Rakotondrabe \\ Automatic Control and MicroMechatronic Systems (AS2M) department \\ FEMTO-ST Institute \\ CNRS UMR-6174, University of Franche-Comté, ENSMM, UTBM \\ 24, rue Alain Savary Besançon France; \\ rkanty@femto-st.fr; mrakoton@femto-st.fr
}

\begin{abstract}
Mesoscale robots, including active capsules, are a promising and well suited approach for minimal invasive intrabody intervention. However, within the numerous works, the main limitation in these robots is the embedded energy used for their locomotion and for the tasks they should accomplish. The limited autonomy and the limited power make them finally unusable for real situations such as active capsules inside body during several tens of minutes. In this paper, we propose an approach to power mesoscale robots by using energy harvesting techniques through a piezoelectric cantilever structure embedded on the robot and through an oscillating magnetic excitation. The physical model of the proposed system is carried out and simulation results are yielded and analyzed accordingly to the influencing parameters such as the number of layers in the cantilever and its dimensions. Finally, the feasability of this solution is proved and perspectives are discussed.
\end{abstract}

Keywords: Capsules, energy harvesting, magnetic excitation, piezoelectric harvester, mesoscale robots.

\section{INTRODUCTION}

Mesoscale robots and active capsules are clearly identified as future trends of medical robots thanks to their dimensions more suitable for minimal invasive intervention. Among the applications of mesorobots which are essentially capsules, we find: imaging of gastrointestinal tract, biopsy, ${ }^{1}{ }^{2}$ The most successful passive capsule in the literature has been used to achieve monitoring or video acquisition inside a small bowel or GI tract. ${ }^{3}$ Nowadays, the development of active capsules is continuously increasing. The main limitation of active capsules, however, is the embedded energy used both for locomotion and for task accomplishment ${ }^{4,5}$ despite of the multipurpose robotic abilities. ${ }^{3,6}$ This energy limitation is particularly due to the miniature volume of the capsule and thus a difficulty to embedd accumulators within this latter.

The use of a direct energy transfer from an external source based on magnetic interaction to move the nano, micro or mesorobots has been investigated. ${ }^{7-9}$ Their limitation is mainly the reduced achievable functions as navigation or video acquisition. Further, another energy transfer was proposed to power embedded motors by using inductive coils. ${ }^{4,10}$ However, this solution suffers from the limited efficiency of the transmitted energy (less than 5\%) and from the non-conformity with the SAR $=0.4 \mathrm{~W} / \mathrm{Kg}$ (Specific Absorption Rate) prescribed by the ICNIRP (International Commission on Non-Ionizing Radiation Protection) and with the working frequency recommanded for human bodies applications. ${ }^{11}$ Indeed, the frequency range has to be less than $100 \mathrm{KHz}$ in order to avoid significant absorption of energy and temperature increase.

In order to improve the capability of mesorobots, we propose an alternative solution based on energy harvesting system embedded in the mesorobot. Although several materials (piezoelectric, magnetostrictive, and electroactive polymer, etc.) can be employed to convert mechanical energy to electric one, piezoelectric materials are undeniably the most appreciated for such applications thanks to the high retrievable energy density for a given size ${ }^{12-14}$ or if the structure is well designed. ${ }^{15}$ In addition, the literature is mainly focused on a vibration energy harvesting for Wireless Sensor Network (WSN). ${ }^{16-18}$

In this paper, we propose a system to overcome the intracorporeal mesorobot limitation of autonomy through energy harvesting. Based on an external oscillating magnetic excitation and a piezoelectric structure, the proposed approach can be used to power capsules mesorobots autonomous for locomotion and for intra-body tasks 
accomplishments. The paper is organized as follows. The proposed design for harvesting system is first described in section II. Section 3 is devoted to the presentation and modeling of the magnetic excitation. In section IV, the piezoelectric system for the harvesting is presented and modeled while section $\mathrm{V}$ is devoted to the simulation results and discussion. Finally, conclusions and perspectives are discussed in section 7 .

\section{PRESENTATION OF THE WHOLE HARVESTING MODULE}

The harvesting module is a part of the mesorobot capsule. The harvester module was designed according to mesoscale robot dimensions in the literature. Typical existing capsules allow us to design a harvesting module enclosed in a cylinder with about $10 \mathrm{~mm}$ diameter and $40 \mathrm{~mm}$ length. In figure 1-(a) is shown a Solidworks-CAD scheme of the module. The module has the following elements.

- A cantilever based on piezoelectric material is the basis of the harvesting system. When bended, charge appears on the surface of the cantilever (accordingly to the direct piezoelectric principle). This charge can be afterwards transformed into voltage $\left(V^{+}-V^{-}\right)$to power the mesorobot capsule thanks to an electric circuit. A spherical magnetic object, called mass proof, is glued at the extremity of the cantilever. Its diameter is imposed to be $1 \mathrm{~mm}, 1.5 \mathrm{~mm}$ or $2 \mathrm{~mm}$ for an ease of fixation with the cantilever.

- A magnetic source is used as excitation to bend the piezoelectric cantilever through the magnetic object. The magnetic field to be used is oscillating with a frequency nearly similar to the first resonant frequency of the piezoelectric cantilever. At this frequency, the deflection of the latter is high and consequently the yielded charge is important. We assume that it is always possible to orient the external excitation system such that the direction of the magnetic field is parallel to the deflection of the cantilever ( $Z$-axis). This assumption can be satisfied easily since the harvester module orientation can be controlled by embedded actuators.

- the cantilever is clamped and enclosed in the magnetically permeable cylindrical package as seen in figure $1-(b)$.
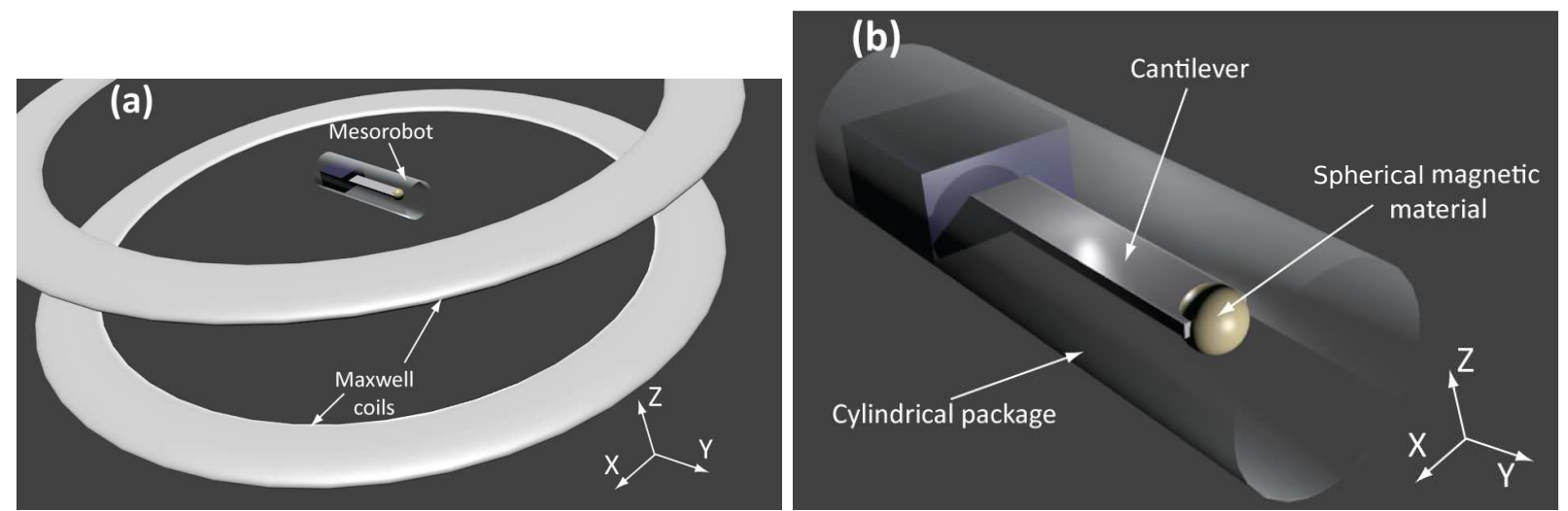

Figure 1. (a) Principle of the harvesting system for mesoscale robot in the constant gradient zone and (b) energy harvester module of the mesorobot.

It is worth to remark that we also use magnetic field because of its biocompatibility relative to other excitation principles (thermal, etc). In addition, its ability has been demonstrated in some medical applications:

- a robot manipulator that interacts with a capsule and that is based on a permanent magnet for gerenating displacement, ${ }^{3}$

- a modified MRI for micro/mesorobot ${ }^{7}$ and actuator for needle insertion, ${ }^{19}$

- other Saddle/Maxwell coil arrangement,${ }^{20}$

- solenoide arrangement for intraocular microrobot. ${ }^{21}$ 


\section{EXCITATION SYSTEM MODELING}

In this section, we model the magnetic excitation system in order to provide an analytical formulation of the interaction force with the piezoelectric cantilever and the magnet.

\subsection{Expression of the Magnetic Force versus the gradient and Mass Proof}

We consider that the capsule stays in a zone with constant magnetic gradient. The mass proof is a soft magnetic body $\left(V_{p}\right)$ which interacts to the magnetic field $B$. It is possible to drive a soft magnetic body in multi-DOF ${ }^{15,20,21}$ but this study is limited to one DOF in order to demonstrate the effectiveness of the proposed harvesting system. Thus the force along $\mathrm{Z}$ is derived as:

$$
F_{z}=n_{d} V_{p} M_{s z} \frac{\partial B}{\partial z}
$$

where $n_{d}$ is the duty cycle, $V_{p}$ is the volume of the active material and $M_{s z}$ is the magnetization.

\subsection{Magnetic source modeling}

In medical application, the robot has to stay in a zone with constant magnetic gradient and with controllable magnetic field. This requirement can be fulfilled easily by adjusting the design. In fact, recent works have shown that Maxwell coils are able to generate a constant gradient in a specific design. ${ }^{20}$ Therefore, by using two Maxwell coils, the expression of the generated magnetic field is:

$$
B=\mu_{0} N R I^{2}\left[\frac{1}{2\left(\left(z-\frac{\alpha R}{2}\right)^{2}+R^{2}\right)}-\frac{1}{2\left(\left(z+\frac{\alpha R}{2}\right)^{2}+R^{2}\right)}\right]
$$

where $\alpha$ defines the ratio between the axial distance $D$ of two coils and their radius $R, I$ is the electric current, and $N$ is the number of turns. Figure 2 shows the variation of magnetic field and gradient for $\alpha=1.9$ : the maximum gradient is $29.3 \mathrm{mT} / \mathrm{m}$ and $5 \%$ of variation is obtained between $-0.25 \mathrm{~m}$ to $0.25 \mathrm{~m}$. This zone is large enough to contain a human part: abdomen, head, leg, etc. By decreasing the radius of the coil (and also the distance D), it possible to get higher values of the gradient as observed in. ${ }^{22}$ The drawback is the reduction of the workspace with constant gradient.
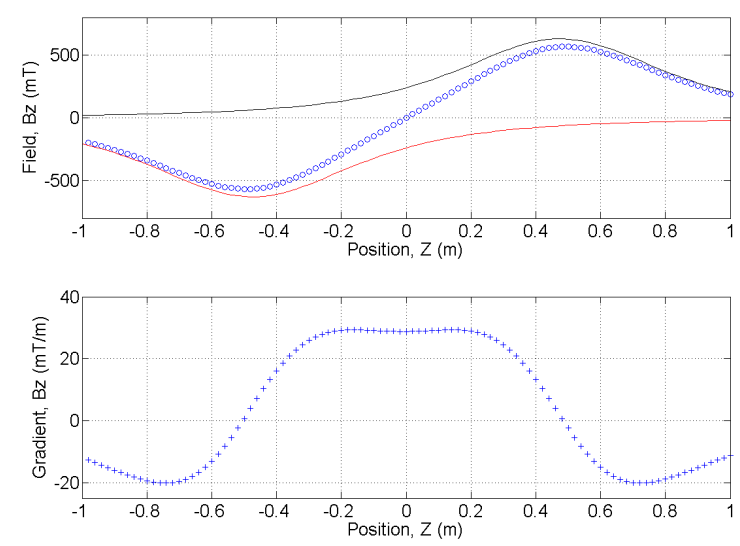

Figure 2. Variation of magnetic field and its gradient for $\alpha=1.9, \mathrm{R}=0.5 \mathrm{~m}, \mu_{0}=1.26 \mathrm{e}^{-6}, \mathrm{I}=100 \mathrm{~A}$, and $\mathrm{N}=5000$.

\subsection{Gradient and force range in medical applications}

In the literaturen, there were some results about the use of magnetic excitation to move ferromagnetic or soft magnetic bodies in medical applications (see Tab 1). The main issues referred to the maximum value of the gradient, the maximal/minimal volume of the movable soft magnetic body and the force range. In fact, none of these previous works can provide the requirement for the proposed capsule in term of field gradient, force range 
(see figure 2) and volume and shape of the mass proof expected in our applications. However, based on the model and analysis in 3.1 and 3.2 and on figure 2, we can derive the interaction force versus the gradient as pictured in figure 3 , and we can observe from this that it is possible to obtain a force around $1 \mathrm{mN}$ by appropriately choosing the gradient and/or the radius of the magnetic body. Consequently, it is possible to design a harvester that will satisfy our requirements.

Table 1. Magnetic excitation parameters for meso/microrobots in medical applications

\begin{tabular}{|l|l|l|l|l|}
\hline Reference & $\begin{array}{l}\text { Gradient } \\
(\mathrm{mT} / \mathrm{m})\end{array}$ & Dimension $(\mathrm{mm})$ & $\begin{array}{l}\text { Magnetization } \\
\left(10^{6} \mathrm{~A} / \mathrm{m}\right)\end{array}$ & Force $(\mathrm{mN})$ \\
\hline${ }^{23}$ & 700 & ellipse $0.95 \times 0.4$ & 0.5 & 0.009 \\
\hline 7 & 40 & sphere $r=0.75$ & 1.35 & 0.85 \\
\hline${ }^{24}$ & 310 & ellipse $4.9 \times 2.67$ & 0.61 & 250 \\
\hline${ }^{21}$ & - & $0.8 \times 0.8 \times 1.2$ & 0.5 & 0.11 \\
\hline 19 & 40 & sphere $r=2.5$ & 1.36 & 1200 \\
\hline
\end{tabular}

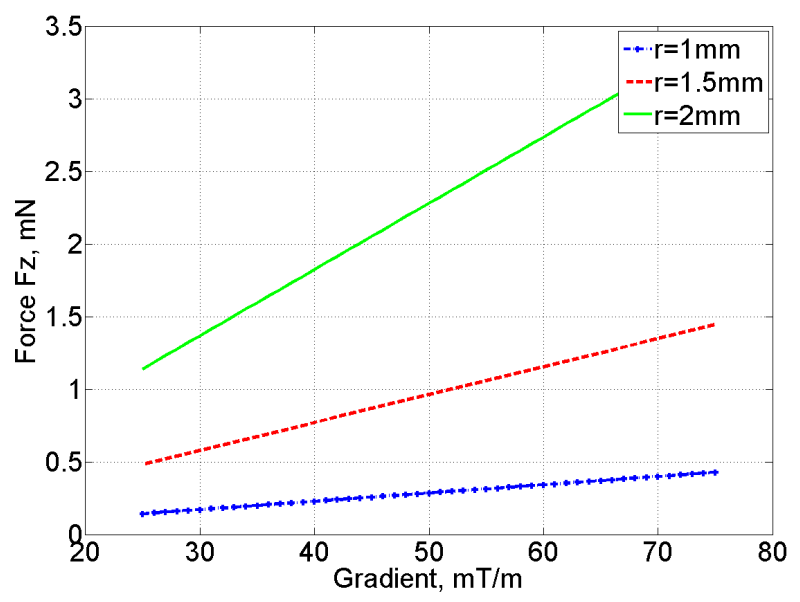

Figure 3 . Force $F_{z}$ versus the gradient variation with three radius of magnetic body $\left(M_{s z}=1.3610^{6} \mathrm{~A} / \mathrm{m}\right)$.

\section{MODELING THE MULTIMORPH PIEZOELECTRIC CANTILEVER WITH MASS PROOF}

In the previous section, we provided the model of the magnetic excitation and we derived an order of value of the magnetic force that acts on the mass proof. In this section, we give the analytical model of the piezoelectric cantilever combined with the mass proof, the objective being to yield the output electricity (in term of charge $Q)$ and the deflection of the cantilever versus the excitation force.

\subsection{Presentation of the structure}

In order to characterize the optimal structure that would provide the best performances in term of energy harvesting, we consider the general case of a piezoelectric cantilever with $n$ layers. The layers can be of piezoelectric material (called piezolayers) or non-piezoelectric material (called passive layer). A cantilever with $n$ layers (piezo and passive) where the number of piezolayers is $n_{p}\left(n_{p} \leq n\right)$ is called $n_{p}$-morph cantilever and has a $n$-layered structure. $^{25,26}$ For instance, a unimorph with uni-layered cantilever has only one piezolayer (Fig. 4-b); a unimorph bi-layered cantilever has two layers made up of one piezolayer and one passive layer (Fig. 4-a); a bimorph cantilever with 2-layers is made up of two piezolayers (Fig. 4-c); etc. Consider the multimorph piezoelectric cantilever that supports the mass proof as pictured in Fig. 4-a. The active length of the cantilever is denoted $l$, the 
widths of the different layers are imposed to be constant and equal to $w$, the thickness of the $i^{\text {th }}$ layer is denoted $h_{i}$ such that the total thickness is $h=\sum_{i=1}^{n} h_{i}$. The length $l$ of the cantilever is infinitely high relative to the total thickness $h$. It results that the diameter of the mass proof is negligible face to the length of the cantilever as this diameter is in the same order than the thickness. Consequently, we can assume that the application of a force on the object comes to the application of the force at the tip of the cantilever. The application of an external force $F_{z}$ at the tip or a moment $M$ about the out-of-plane $y$-axis will result in a deflection $\delta_{z}$ of the cantilever and in an apparition of a total charge $Q$ at the different electrodes of the piezolayers (Fig. 4-b). Remark that the local $z$-axis of the cantilever is parallel to the $Z$-axis of the magnetic field.

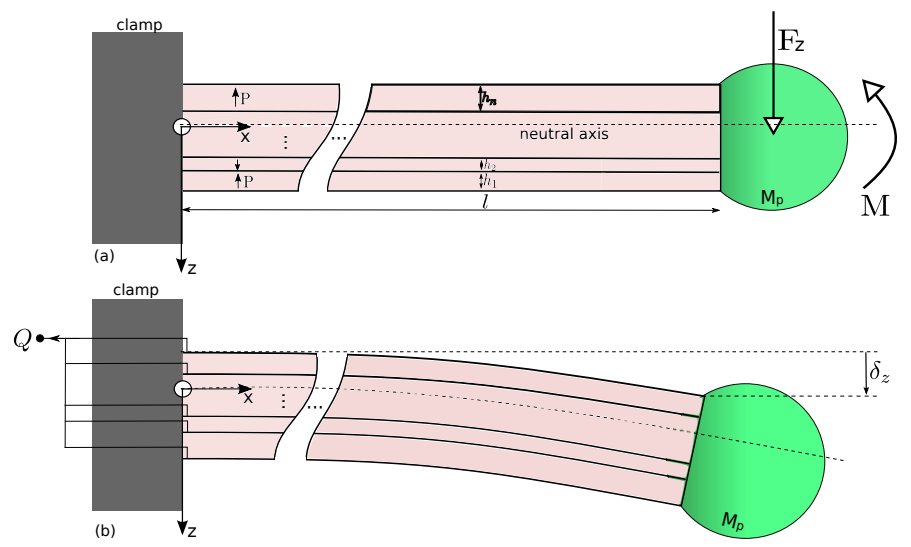

Figure 4. (a): a multimorph piezoelectric cantilever with a mass proof at its extremity and with an external force and moment as excitation. (b): the resulting deflection $\delta_{z}$ and resulting total charge $Q$.

\subsection{Governing equations of the multimorph piezoelectric cantilever without mass proof}

In this subsection, we derive the equations of the deflection $\delta_{z}$ and of the charge $Q$ when applying a harmonic force $F_{z}(t)$ and a harmonic moment $M(t)$. We consider that the mass proof is first absent. The model of the piezoelectric cantilever when the stationary regime is reached can be derived form the general from in ${ }^{25}$ by studying the deflection at the tip (distance $l$ ) of the cantilever and by focusing the analysis on the first resonant mode. We obtain:

$$
\left(\begin{array}{c}
\delta_{z}(L, t) \\
Q(L, t)
\end{array}\right)=\left(\begin{array}{ll}
Z_{11} & Z_{12} \\
Z_{21} & Z_{22}
\end{array}\right)\left(\begin{array}{c}
F_{o} \cos (\Omega t-\psi) \\
M_{o} \cos (\Omega t-\psi)
\end{array}\right)
$$

where $Z=\left(\begin{array}{cc}Z_{11} & Z_{12} \\ Z_{21} & Z_{22}\end{array}\right)$ is the transmittance (matrix), $F_{o}$ (resp. $M_{o}$ ) is the amplitude of the harmonic force (resp. moment) and $\Omega$ is the frequency $(\mathrm{rad} / \mathrm{s})$. Notice that the force $F_{z}$ calculated in section III is used as the amplitude $F_{o}$ of the force here. The moment $M_{o}$ is such that: $M_{o}=F_{o} \cdot l$. We have:

$$
\left\{\begin{array}{l}
Z_{11}=\frac{4}{m} \frac{\Gamma_{1} \alpha_{F}}{\omega^{2} \sqrt{\left(1-\eta^{2}\right)^{2}+(2 \zeta \eta)^{2}}} \\
Z_{12}=-\frac{4}{m} \frac{\Gamma_{1} \alpha_{M} k}{\omega^{2} \sqrt{\left(1-\eta^{2}\right)^{2}+(2 \zeta \eta)^{2}}} \\
Z_{21}=-\frac{4}{m} \frac{\Gamma_{2} \alpha_{F} c_{p}}{\omega^{2} \sqrt{\left(1-\eta^{2}\right)^{2}+(2 \zeta \eta)^{2}}} \\
Z_{22}=\frac{4}{m} \frac{\Gamma_{2} \alpha_{M} c_{p} k}{\omega^{2} \sqrt{\left(1-\eta^{2}\right)^{2}+(2 \zeta \eta)^{2}}}
\end{array}\right.
$$

where $\zeta<1$ is the attenuation coefficient (damping coefficient) and $m$ is the mass of the piezoelectric cantilever and coefficient $\frac{m}{4}$ in (Eq.4) indicates that the model was derived considering a quarter of $m$ as mass seen at the tip of the cantilever. A precise calculation of mass $m$ is based on the geometric properties of the different layers 
and on their densities $\rho_{i}: m=w l \sum_{i=1}^{n} \rho_{i} h_{i}$. Coefficient $\eta$ is the ratio between the excitation frequency $\Omega$ and the undamped oscillation $\omega: \eta=\frac{\Omega}{\omega}$, such that $\omega=\frac{(k l)^{2}}{l^{2}} \sqrt{\frac{C l}{m}}$, where $k l=1.8751$ for the considered first mode and where the flexure rigidity $C$ is defined by:

$$
C=\frac{w}{3} \sum_{i=1}^{n} \frac{1}{s_{11, i}}\left[3 h_{i}\left(\bar{z}-\sum_{j=1}^{i} h_{j}\right)\left(\bar{z}-\sum_{j=1}^{i-1} h_{j}\right)+h_{i}^{3}\right]
$$

$\bar{z}$ being the distance of the neutral axis from the lower surface of the cantilever and is defined by:

$$
\bar{z}=-\frac{\sum_{i=1}^{n} \frac{h_{i}^{2}}{s_{11, i}}-2 \sum_{i=1}^{n} \frac{h_{i}}{s_{11, i}} \sum_{j=1}^{i} h_{j}}{2 \sum_{i=1}^{n} \frac{h_{i}}{s_{11, i}}}
$$

Coefficient $s_{11, i}$ is the elastic coefficient of the $i^{\text {th }}$ layer. If the layer is a non-piezoelectric material (passive layer), the elastic coefficient corresponds to: $s_{11, i}=\frac{1}{E_{11}}$, with $E_{11}$ being the axial elasticity (Young modulus along $x$-axis). We also have:

$$
c_{p}=\frac{w}{2} \sum_{i=1}^{n} \frac{d_{31, i}}{s_{11, i} h_{i}}\left[2 \bar{z} h_{i}-2 h_{i} \sum_{j=1}^{i} h_{j}+h_{i}^{2}\right]
$$

where $d_{31, i}$ is the trransversal piezoelectric coefficient of the $i^{\text {th }}$ piezolayer. If the layer is passive, i.e. nonpiezoelectric material, we have: $d_{31, i}=0$.

The remaining coefficients of (Eq.4) are described in Tab 2

Table 2. Parameters for the transmittance $Z$.

\begin{tabular}{|l|l|}
\hline \hline Parameter & Name \\
\hline$\Psi=\arctan \left(\frac{2 \zeta \eta}{1-\eta^{2}}\right)$ & phase \\
\hline $\begin{array}{l}\alpha_{F} \cosh (k l) \sin (k l)-\cos (k l) \sinh (k l) \\
\sin (k l)+\sinh (k l)\end{array}$ & \\
\hline$\alpha_{M}=\frac{\sin (k l) \sinh (k l)}{\sin (k l)+\sinh (k l)}$ & \\
\hline$\Gamma_{1}=\tilde{c}-\tilde{s} \frac{C}{\tilde{S}}$ & the eigenmode \\
\hline$\Gamma_{2}=k\left(\tilde{S}-\tilde{c} \frac{\tilde{C}}{\tilde{S}}\right)$ & \\
\hline$\tilde{S}=\frac{1}{2}(\sinh (k l)+\sin (k l))$ & \\
\hline $\begin{array}{l}\tilde{C} \\
\frac{1}{2}(\cosh (k l)+\cos (k l))\end{array}$ & \\
\hline$\tilde{s}=\frac{1}{2}(\sinh (k l)-\sin (k l))$ & \\
\hline$\tilde{c}=\frac{1}{2}(\cosh (k l)-\cos (k l))$ & \\
\hline
\end{tabular}

\subsection{Governing equations of the cantilever with mass proof}

The different elementary transmittances $Z_{i j}$ in (Eq.4) were derived when there is no additional mass along or on the piezoelectric cantilever ${ }^{25}$. This calculation was based on the quarter of the mass $m$ of the cantilever as load at its tip, i.e. $\frac{m}{4}$. However, in our case, there is a mass proof $M_{p}$ at this tip. So, the total mass seen by an excitation at the tip is $\frac{m}{4}+M_{p}$. Consequently, instead of using (Eq.4), the final transmittances are: 


$$
\left\{\begin{array}{l}
Z_{11}=\frac{1}{\left(\frac{m}{4}+M_{p}\right)} \frac{\Gamma_{1} \alpha_{F}}{\omega^{2} \sqrt{\left(1-\eta^{2}\right)^{2}+(2 \zeta \eta)^{2}}} \\
Z_{12}=-\frac{1}{\left(\frac{m}{4}+M_{p}\right)} \frac{\Gamma_{1} \alpha_{M} k}{\omega^{2} \sqrt{\left(1-\eta^{2}\right)^{2}+(2 \zeta \eta)^{2}}} \\
Z_{21}=-\frac{1}{\left(\frac{m}{4}+M_{p}\right)} \frac{\Gamma_{2} \alpha_{F} c_{p}}{\omega^{2} \sqrt{\left(1-\eta^{2}\right)^{2}+(2 \zeta \eta)^{2}}} \\
Z_{22}=\frac{1}{\left(\frac{m}{4}+M_{p}\right)} \frac{\Gamma_{2} \alpha_{M} c_{p} k}{\omega^{2} \sqrt{\left(1-\eta^{2}\right)^{2}+(2 \zeta \eta)^{2}}}
\end{array}\right.
$$

\section{SIMULATION AND DISCUSSION}

We simulate in this section the model in (Eq.3) and in (Eq.8) in order to analysis the influence of the geometrical properties on the charge $Q$ and on the deflection $\delta_{z}$. The simulation analysis was carried out using MatlabSimulink software. The different configurations of the cantilever used for the simulation are illustrated in Fig. 4. We have:

- a unimorph 2-layered piezoelectric cantiliver (piezocantilever),

- a unimorph 1-layered piezocantilever,

- a bimorph piezocantilever,

- and a $n$-morph $(n=3 \cdots 10)$ piezocantilever.

The piezoelectric material proposed for this application is the monocrystal PMN-PT (lead magnesium niobate - lead titanate). Its principal advantages are the high coupling factor relative to piezoelectric materials commonly used (such as PZT). Another advantage of the PMN-PT is its compatibility with microfabrication techniques (DRIE, laser, cutting, etc.) in particular when combined with Silicone material. ${ }^{27}$ This latter advantage is essential for the packaging aspect because it is possible to fabricate the systems as small as the microfabrication technique is able to do. Though we use PMN-PT in the simulation, other materials $(\mathrm{PZT}, \ldots)$ can be used for the model and for the simulation. The numerical values of the used parameters are summarized in Tab. 3.

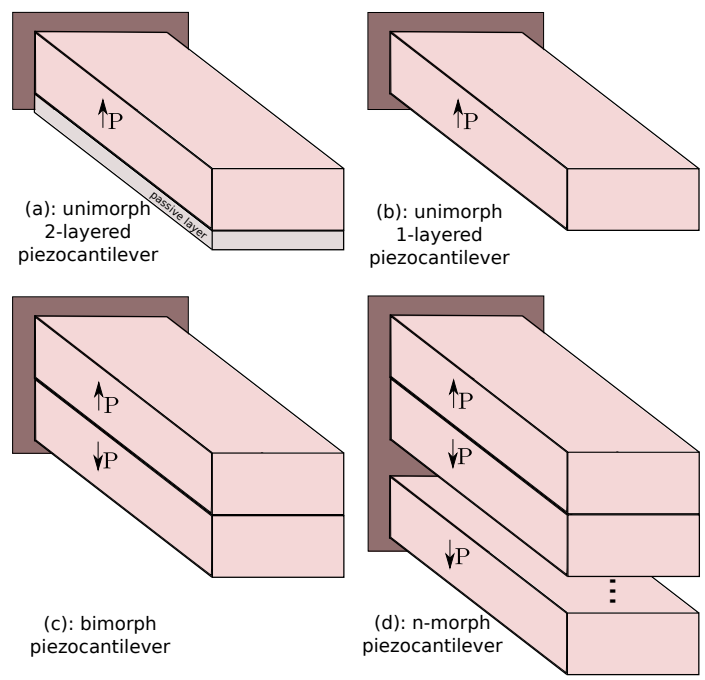

Figure 5. Different possible piezocantilevers (without mass proof). 
Table 3. Numerical values for the simulations.

\begin{tabular}{|l|l|}
\hline \multicolumn{2}{|c|}{ Cantilever dimensions: } \\
\hline \hline$l$ & $15 \mathrm{~mm}, 25 \mathrm{~mm}, 35 \mathrm{~mm}$ \\
\hline$w$ & $2 \mathrm{~mm}, 4 \mathrm{~mm}, 6 \mathrm{~mm}$ \\
\hline$h_{p z t}$ & $\begin{array}{l}\text { see values in the Fig- } \\
\text { ures }\end{array}$ \\
\hline$h_{s i}$ & $\begin{array}{l}0.2 \mathrm{~mm} \text { for configura- } \\
\text { tion unimorph 2-layers }\end{array}$ \\
\hline \hline \multicolumn{2}{|c|}{ Excitation: } \\
\hline \hline$F_{0}$ & $1 \mathrm{mN}$ \\
\hline$M_{0}$ & $F_{0} \cdot l$ \\
\hline Mass proof $\left(M_{p}\right)$ & $1 \mathrm{mg}$ \\
\hline \hline Properties of the piezoelectric material PMN-PT: \\
\hline \hline$\rho_{\text {piezo }}$ & $8200 \mathrm{Kg} / \mathrm{m}^{3}$ \\
\hline$s_{11}$ & $60 \mathrm{e}-12 \mathrm{TPa}$ \\
\hline$d_{31}$ & $-1200 \mathrm{e}-12 \mathrm{pm}^{-1} \mathrm{~V}$ \\
\hline$\zeta$ & 0.01 \\
\hline \hline \multicolumn{2}{|c|}{ Properties of the silicone: } \\
\hline \hline$\rho_{S i}$ & $2330 \mathrm{Kg} / \mathrm{m}^{3}$ \\
\hline$s_{11 S i}$ & $1 / 107 \mathrm{e} 9 \mathrm{~m}^{2} / \mathrm{N}$ \\
\hline$d_{31 S i}$ & 0 \\
\hline
\end{tabular}

\subsection{Effect of the number of layers}

The influence of the number of layers on the harvester performances is first investigated. In this investigation, the thicknesses $h_{i}(i=1 \cdots n)$ of the piezolayers are imposed to be equal such that the total thickness $h=\sum_{i=1}^{n} h_{i}$ is increasing with the number $n$ of layers. The results are shown in Fig. 6 with different possible values of thickness $h_{i}$ tested $\left(h_{i}=0.2 \mathrm{~mm}, h_{i}=0.3 \mathrm{~mm}\right.$ and $\left.h_{i}=0.4 \mathrm{~mm}\right)$. We can observe that the unimorph with one layer provides very negligible charge. In fact, this is due to the absence of (or to the weak) strain along the thickness (expansion/contraction) of the piezolayer, even if the whole cantilever performs high deflection (Fig. $6-b)$. This weak strain along the thickness comes from the fact that the surfaces (upper and lower) of each layer are weakly constrained. We also observe that a lower value of thickness is favorable to obtain a higher charge. Finally, it is deduced from the figure that the furnished charge decreases with the number of layers; and the most interesting configuration in this investigation is the bimorph structure (2-layers).

In the second investigation, we impose the total thickness $h$ to be constant whatever the number $n$ of layers is. The results are pictured in Fig. 7. On the one hand, the deflection is independant from the number of layers except for the unimorph 1-layer case. This is predictible since the stiffness is unchanged when the total thickness $h$ is left constant when the all layers that compose the cantilever are the same (PMN-PT only). On the other hand, once again we find that the charge is increased when the thickness $h$ (and consequently the elementary thickness $h_{i}$ ) is decreased. It is tempting to choose layers and cantilever with weak thickness. Meanwhile, they are fragile and very difficult to fabricate. In addition, the cantilever has to be rigid enough to support the mass proof.

\subsection{Effect of the cantilever length and width}

Investigation on the cantilever length and width effect is now performed. The results are pictured in Fig. 8. We can note that by increasing the width $w$ of the length $l$, we increase the obtained charge. This is due to the fact that by increasing these two parameters will increase the electrodes surfaces $l \cdot w$, and therefore will increase the charge itself. The main limitation to having these parameters high lies on the restricted available volume of the capsule. 

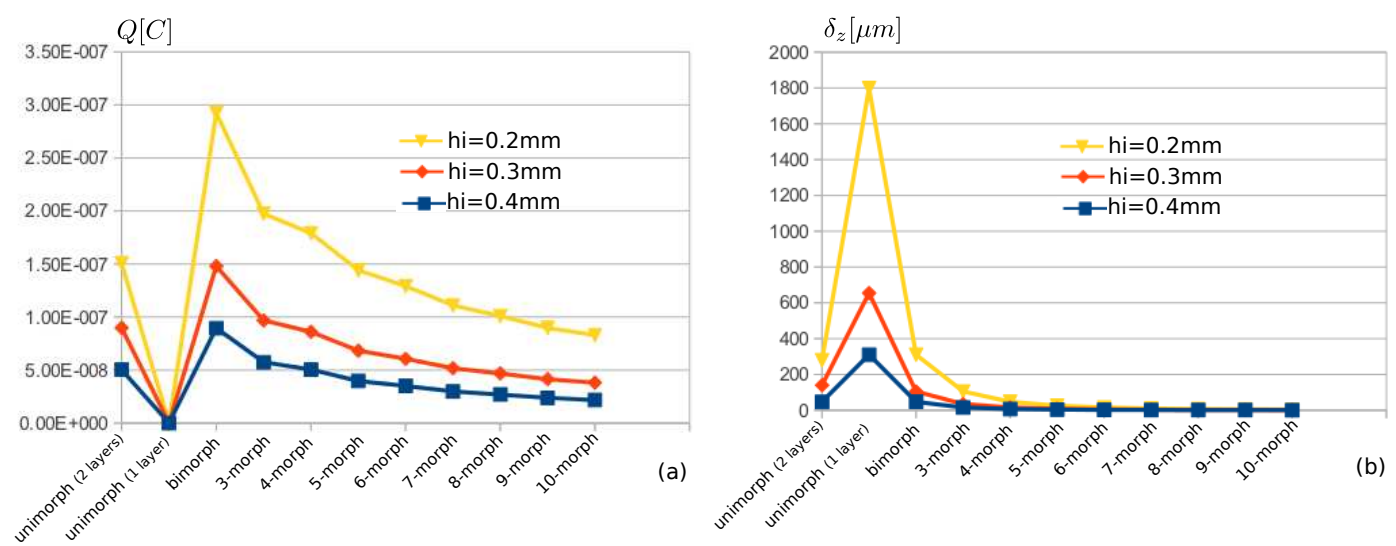

Figure 6. (a): evaluation of charge $Q[C]$ versus the number of layers $n$ for a given thickness $h_{i}$ of the piezolayer(s). (b): evaluation of the deflection $\delta_{z}[\mu \mathrm{m}]$ versus the number of layers $n$ for a given thickness $h_{i}$ of the piezolayer(s).
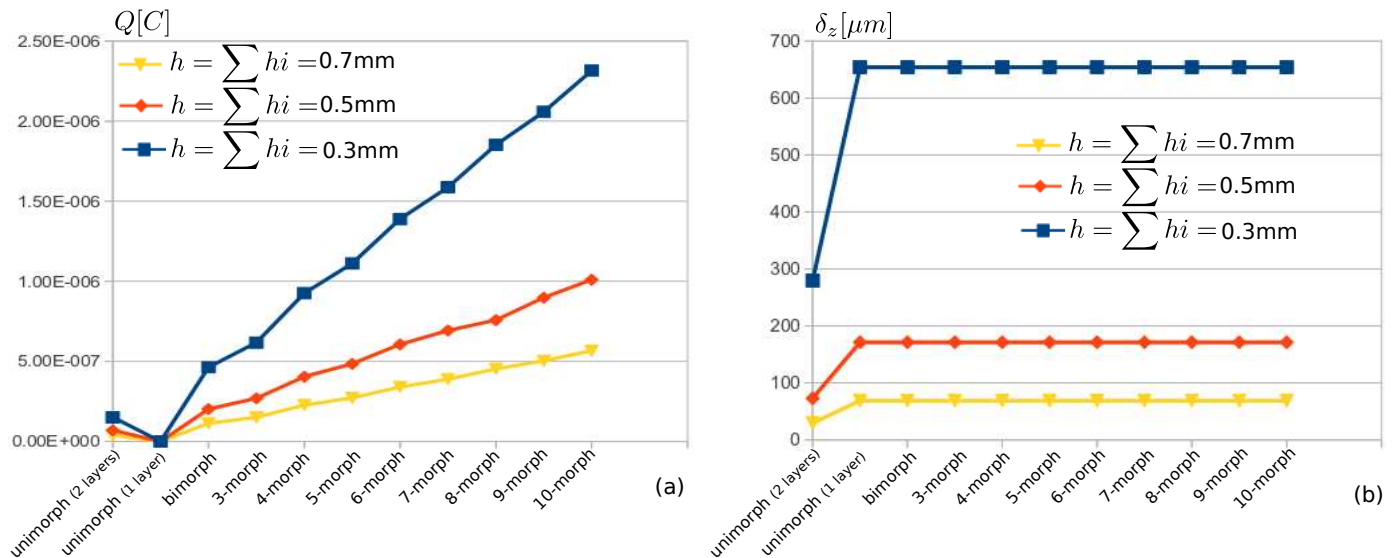

Figure 7. . (a): evaluation of charge $Q[C]$ versus the number of layers $n$ for a given value of the total thickness $h$ of the cantilever. (b): evaluation of the deflection $\delta_{z}[\mu \mathrm{m}]$ versus the number of layers $n$ for a given value of the total thickness $h$ of the cantilever.
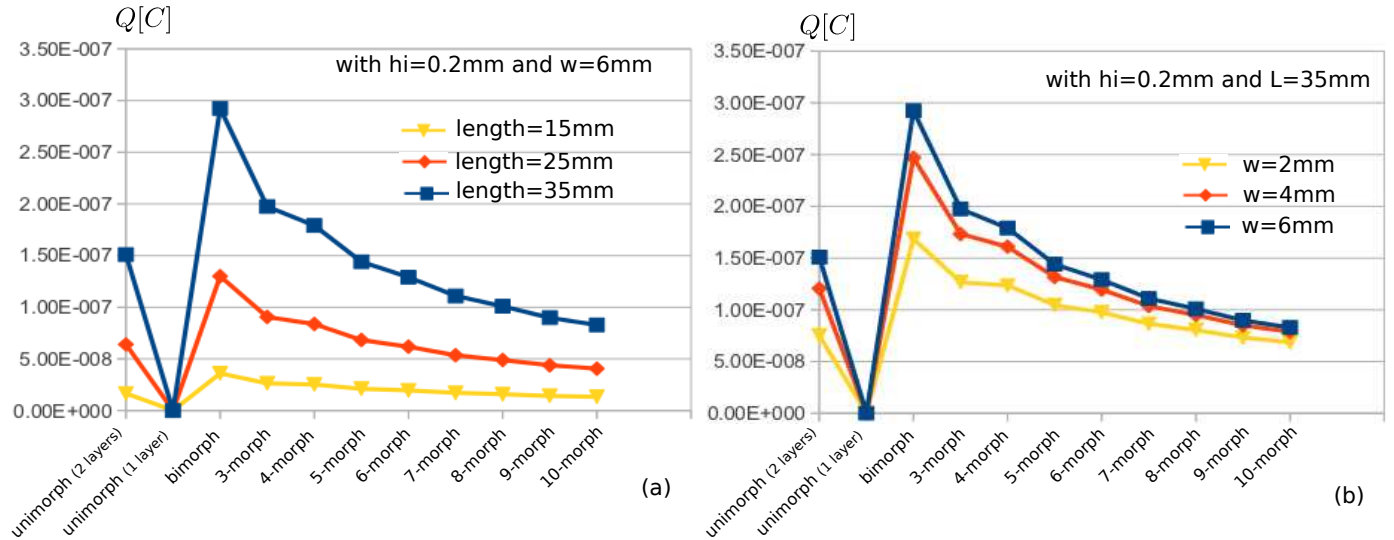

Figure 8. (a): evaluation of charge $Q[C]$ versus the number of layers $n$ for a given length $l$. (b): evaluation of charge $Q[C]$ versus the number of layers $n$ for a given width $w$.

\subsection{Variation of the resonance frequency}

The resonance frequency variation is also studied according to the number of layer and for a fixed thickness $h_{i}=0.2 \mathrm{~mm}$ of piezolayers. We can observe in Fig. 9 that the frequency increases with the number of layer. Furthermore, the longest cantilever $L=35 \mathrm{~mm}$ provides the lowest resonance frequency. The study of the 
resonance frequency is essential because it is directly linked to the ability of the excitation system. In fact, high frequency needs special design of the electronic part due to the risk of overheating.

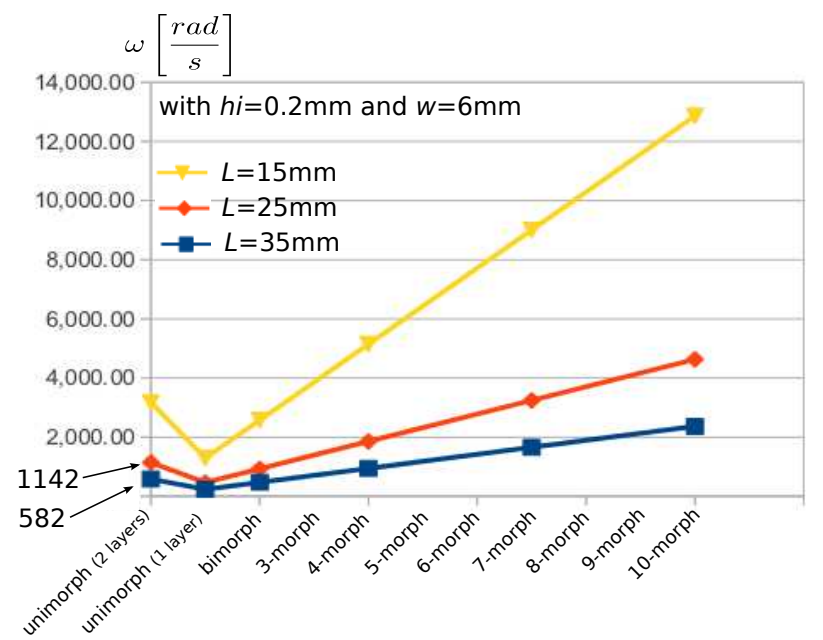

Figure 9. Evaluation of the frequency $\omega[\mathrm{rad} / \mathrm{s}]$ versus the number of layers $n$ for a given length.

\section{PROOF OF CONCEPT}

A prototype is tested to prove the feasibility of the concept. It is based on Macro Fiber Composite (MCF) from SMART MATERIAL which is a rectangular piezoelectric ceramic rod sandwiched between layers of adhesive, electrodes and polyamide film. The cantilever can be considered as a unimorph with two layers: one piezoelectric layer and one passive layer. Figure 10-a shows the experimental setup to prove the concept. The setup is composed of:

- the piezoelectric cantilever with a mass proof at its tip. Two different sizes have been used for that. They are presented in Figure 10-b and their characteristics are listed in Table 4 (datas from ${ }^{28}$ ). The dimensions were chosen to be in the order of the simulation ones. Also, an optical sensor is used to measure the displacement (bending) of the cantilever,

- an electromagnet that excites the proof mass. Its alimentation is supplied by a voltage generator and a voltage/current converter,

- an electrical circuit that transforms the charge appearing on the piezoelectric cantilever's electrodes into voltage,

- and an oscilloscope to display the recuperated voltage.

Table 4. characteristics of the piezoelectric cantilevers.

\begin{tabular}{|l|l|}
\hline MCF 1 & $L \times w \times h=28 \times 7 \times 0.3 m m$ \\
$d_{31}$ & $-2.1 \mathrm{e}-10 \mathrm{C} / \mathrm{N}$ \\
\hline MCF 2 & $L \times w \times h=28 \times 14 \times 0.3 \mathrm{~mm}$ \\
$d_{31}$ & $-2.1 \mathrm{e}-10 \mathrm{C} / \mathrm{N}$ \\
\hline
\end{tabular}

The magnetic excitation is controlled to be sinusoidal thanks to the generator, the voltage/current converter and electromagnet. In the meantime the resulting displacement at the cantilever tip is observed, see figure 11Top. The voltage generated by the harvester is measured from the output of the harvester module at different frequencies and with the two MCF. Results are shown on figure 11-Left for MCF 1 after 45 minutes and 


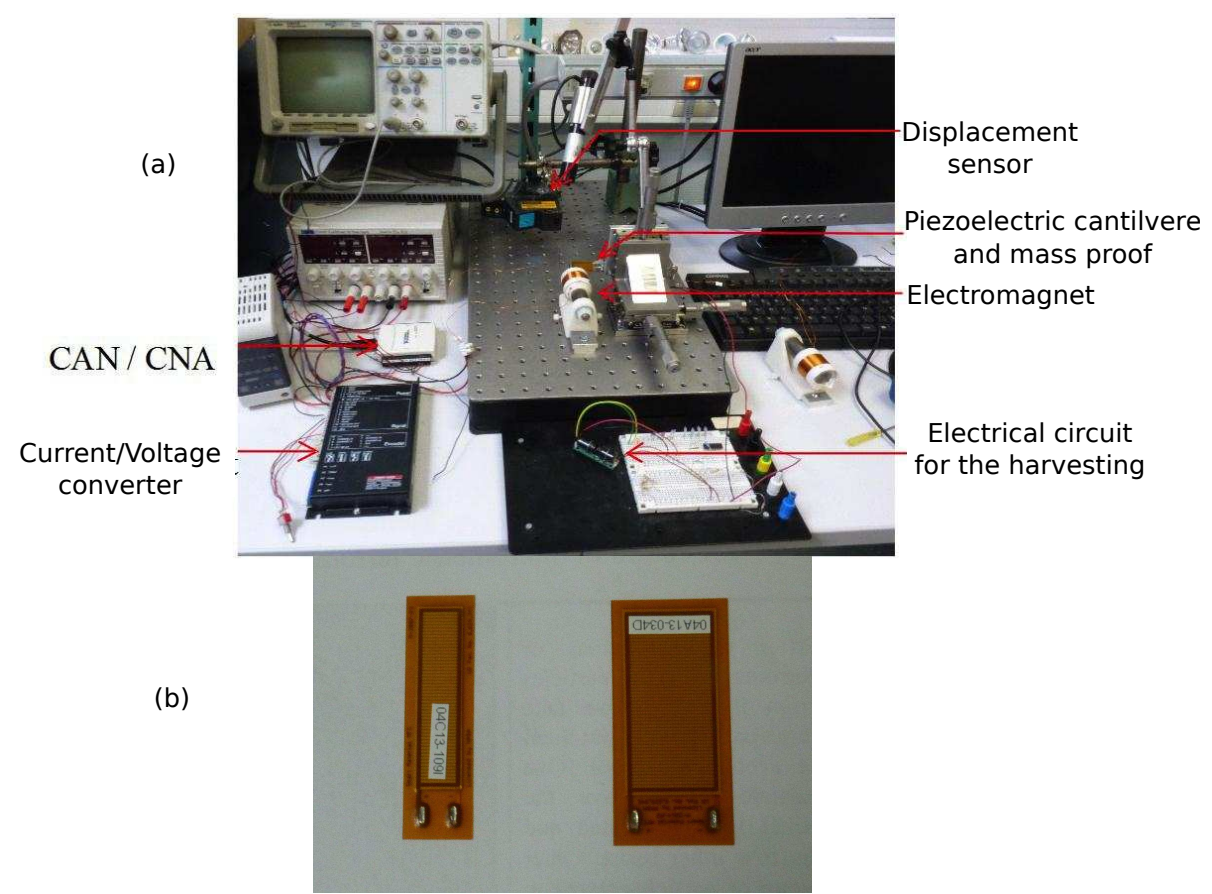

Figure 10. (a) The experimental setup for energy harvesting power mesoscale robot. (b) The used piezoelectric cantilevers.

figure 11-Right for MCF 2 after 30 minutes. First, they clearly confirm that the supplied voltage increases with the excitation frequency, as already demonstrated in the literature. Then, they show that, within the utilized dimensions of piezoelectric cantilevers, the supplied voltage is already promising (some $\mathrm{mV}$ ) even at low frequency (some Hertz). This means that, by choosing a better configuration of the cantilever within the imposed dimensions which are limited by the sizes of the capsule, it is possible to yield higher voltage. For instance, by using a bimorph based on two-layers cantilever, we can maximize the yielded voltage according to the analysis and simulation carried out in the previous sections.

\section{CONCLUSIONS AND PERSPECTIVES}

The in-vivo intervention of mesoscale robots like capsules during MIS (minimal invasive surgery) constitutes the future step of the surgical robotics. It will enable painless and improve maneuverability of tools inside an human body. The approach of energy harvesting is proposed in this paper in order to achieve a wireless power supply of mesoscale robot devited to such objective. The magnetic excitation is chosen thanks to its ability to generate remote and high force, its biocompatibility, and the possibility to use a MRI (magnetic resonance imaging) as an actuator. The harverster module based on piezoelectric material is modeled. Simulation results are presented with thorough analysis according to the number of layer, the thickness of layer, the cantilever length, and the width. They suggested the efficiency of bimorph cantilever compared to the other configurations of piezoelectric cantilever thanks to the following reasons.

\section{ACKNOWLEDGMENTS}

This work is supported by BQR project from University of Franche Comté. It is also supported by the Labex ACTION project (contract ANR-11-LABX-0001-01) and the French RENATECH network and its FEMTO-ST technological facility.

\section{REFERENCES}

[1] Carta, R., Thon, J., and Puers, R., "A wireless power supply system for robotic capsular endoscopes," Sensors and Actuators A: Physical 162, 177-183 (2010). 

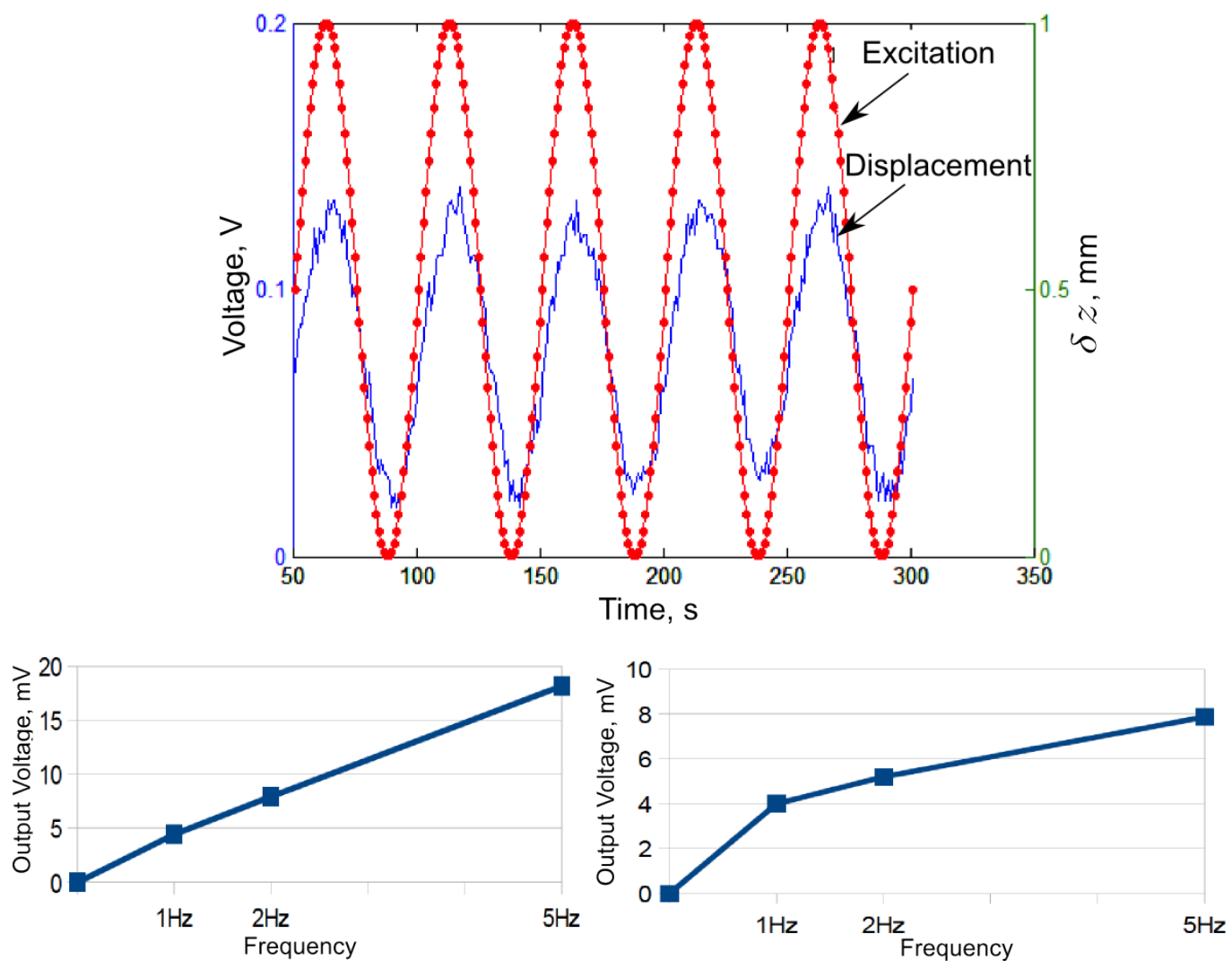

Figure 11. Experimental results of the energy harvesting power mesoscale robot: Top: applied voltage for the excitation and the resulting displacement of the cantilever, Left: output voltage from the harvester with MCF 1 after 45 minutes, and Right: output voltage from the harvester with MCF 2 after 30 minutes.

[2] Quirini, M., Webster, R. J., Menciassi, A., and Dario, P., "Design and fabrication of a motor legged capsule for the active exploration of the gastrointestinal tract," in [IEEE International Conference on Robotics and Automation], (2007).

[3] Moglia, A., Menciassi, A., Schurr, M. O., and Dario, P., "Wireless capsule endoscopy: from diagnostic devices to multipurpose robotic systems," Biomed Microdevices 9, 235243 (2007).

[4] Lenaerts, B. and Puers, R., "An inductive power link for a wireless endoscope," Biosensors and Bioelectronics 22, 1390-1395 (2007).

[5] Dario, P., "Future trends in surgical robotics (part 2)," in [Summer School for Surgical Robotics], (2011).

[6] Toennies, J. L., Tortora, G., Simi, M., Valdastri, P., and R. J. Webster, I., "Swallowable medical devices for diagnosis and surgery: the state of the art," J. Mechanical Engineering Science 224, 1397-1414 (2009).

[7] Martel, S., Mathieu, J.-B., Felfoul, O., Chanu, A., Aboussouan, E., Tamaz, S., and Pouponneau, P., "Automatic navigation of an untethered device in the artery of a living animal using a conventional clinical magnetic resonance imaging system," Applied Physics 90, 1-3 (2007).

[8] Valdastri, P., Quaglia, C., Susilo, E., Menciassi, A., Dario, P., Ho, C. N., Anhoeck, G., and Schurr, M. O., "Wireless therapeutic endoscopic capsule: in vivo experiment," Endoscopy 40, 979-982 (2008).

[9] Park, S., Cha, K., and Park, J., "Development of biomedical microrobot for intravascular therapy," International Advanced Robotic Systems 7, 091-098 (2010).

[10] Carta, R., Pateromichelakis, N., Thon, J., Sfakiotakis, M., Tsakiris, D., and Puers, R., "A wireless powering system for a vibratory-actuated endoscopic capsule," in [Eurosensors XXIV], (2010).

[11] ICNIRP, "Icnirp guidelines for limiting exposure to time-varying electric, magnetic and electromagnetic fields (up to 300 ghz)," Health Physics 74, 494-522 (1998). 
[12] Roundy, S. and Wright, P. K., "A piezoelectric vibration based generator for wireless electronics," Smart Mater. Struct. 13, 1131-1142 (2004).

[13] Sodano, H. A., Inman, D. J., and Park, G., "Comparison of piezoelectric energy harvesting devices for recharging batteries," Journal of Intelligent Material Systems and Structures 16, 799-807 (2005).

[14] A. Bartasyte, M. Rakotondrabe, T. B. and Ballandras, S., "From green piezoelectric materials to designed hybrid piezoelectric energy harvesters," International Workshop on "Advanced Materials Challenges for Alternative Energy Solutions (Incheon Korea, April 2014).

[15] Rakotondrabe, M., "Towards high autonomy energy harvesters based on piezoelectric mems," Nanoenergy, (International Conference on Nanoenergy) (Perugia Italy, July 2013).

[16] Beeby, S. P., Tudor, M. J., and White, N. M., "Energy harvesting vibration sources for microsystems applications," Meas. Sci. Technol. 17, 175-195 (2006).

[17] Ferrari, M., Ferrari, V., Guizzetti, M., Marioli, D., and Taroni, A., "Piezoelectric multifrequency energy converter for power harvesting in autonomous microsystems," Sensors and Actuators A 142, 329-335 (2008).

[18] Zhu, Y., Moheimani, S. O. R., and Yuce, M. R., "A 2-dof mems ultrasonic energy harvester," IEEE Sensors Journal 11, 155-161 (2011).

[19] Vartholomeos, P., Qin, L., and Dupont, P. E., "Mri-powered actuators for robotic interventions," in [IEEE/RSJ International Conference on Intelligent Robots and Systems], (2011).

[20] Han, B. H., Park, S., and Lee, S. Y., "Gradient waveform synthesis for magnetic propulsion using mri gradient coils," Physics in Medecine Biology 53, 4639-4649 (2008).

[21] Kummer, M. P., Abbott, J. J., Kratochvil, B. E., Borer, R., Sengul, A., and Nelson, B. J., "Octomag: An electromagnetic system for 5-dof wireless micromanipulation," IEEE Transactions on Robotics 26, 10061017 (2010).

[22] Mathieu, J.-B. and Martel, S., "Magnetic microparticle steering within the constraints of an mri system: Proof of concept of a novel targeting approach," Biomed Microdevices 9, 801-808 (2007).

[23] Yesin, K. B., Vollmers, K., and Nelson, B. J., "Modeling and control of untethered biomicrorobots in a fluidic environment using electromagnetic fields," International Journal of Robotics Research 25, 527-536 (2006).

[24] Abbott, J. J., Ergeneman, O., Kummer, M. P., Hirt, A. M., and Nelson, B. J., "Modeling magnetic torque and force for controlled manipulation of soft-magnetic bodies," IEEE Transactions on Robotics 23, 1247$1252(2007)$.

[25] Ballas, R. G., [Piezoelectric multilayer beam bending actuators: static and dynamic behavior and aspects of sensor integration], Springer (2007).

[26] Rakotondrabe, M., "Piezoelectric cantilevered structures: modeling control and measurement/estimation aspects," Springer Verlag .

[27] Ivan, I. A., Rakotondrabe, M., Agnus, J., Bourquin, R., Chaillet, N., Lutz, P., Ponot, J.-C., Duffait, R., and Bauer, O., "Comparative material study between pzt ceramic and newer crystalline pmn-pt and pzn-pt materials for composite bimorph actuators," Review on Advanced Materials Science (RAMS) 24, 1-9 (2010).

[28] company, S.-M., "http://www.smart-material.com/MFC-product-main.html\#," 